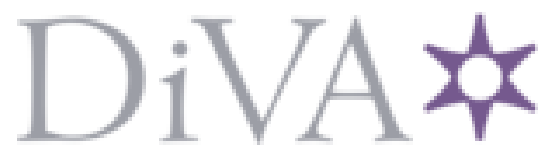

http://www.diva-portal.org

This is the published version of a paper published in Applied Physics Letters.

Citation for the original published paper (version of record):

Huang, S., Vida, A., Li, W., Molnar, D., Kwon, S K. et al. (2017)

Thermal expansion in $\mathrm{FeCrCoNiGa} \mathrm{high-entropy} \mathrm{alloy} \mathrm{from} \mathrm{theory} \mathrm{and} \mathrm{experiment.}$

Applied Physics Letters, 110(24): 241902

https://doi.org/10.1063/1.4985724

Access to the published version may require subscription.

N.B. When citing this work, cite the original published paper.

Permanent link to this version:

http://urn.kb.se/resolve?urn=urn:nbn:se:du-25504 


\section{Thermal expansion in FeCrCoNiGa high-entropy alloy from theory and experiment}

Shuo Huang, Ádám Vida, Wei Li, Dávid Molnár, Se Kyun Kwon, Erik Holmström, Béla Varga, Lajos Károly Varga, and Levente Vitos

Citation: Appl. Phys. Lett. 110, 241902 (2017); doi: 10.1063/1.4985724

View online: http://dx.doi.org/10.1063/1.4985724

View Table of Contents: http://aip.scitation.org/toc/apl/110/24

Published by the American Institute of Physics

\section{Articles you may be interested in}

Tunable electromagnetically induced transparency from a superconducting terahertz metamaterial Applied Physics Letters 110, 241105 (2017); 10.1063/1.4985618

Atomic force microscope based on vertical silicon probes

Applied Physics Letters 110, 243101 (2017); 10.1063/1.4985125

Room temperature magnetodielectric effects in epitaxial hexaferrite $\mathrm{BaFe}_{10.2} \mathrm{Sc}_{1.8} \mathrm{O}_{19}$ thin film Applied Physics Letters 110, 242901 (2017); 10.1063/1.4986438

Enhancement of $\mathrm{L}_{2}$ order and spin-polarization in $\mathrm{Co}_{2} \mathrm{FeSi}$ thin film by substitution of $\mathrm{Fe}$ with $\mathrm{Ti}$ Applied Physics Letters 110, 242401 (2017); 10.1063/1.4985237

Twisted graphene-assisted photocarrier transfer from $\mathrm{HgSe}$ colloidal quantum dots into silicon with enhanced collection and transport efficiency

Applied Physics Letters 110, 241104 (2017); 10.1063/1.4986188

Three-phase $\mathrm{Fe}_{3} \mathrm{O}_{4} / \mathrm{MWNT} / \mathrm{PVDF}$ nanocomposites with high dielectric constant for embedded capacitor Applied Physics Letters 110, 242902 (2017); 10.1063/1.4986443

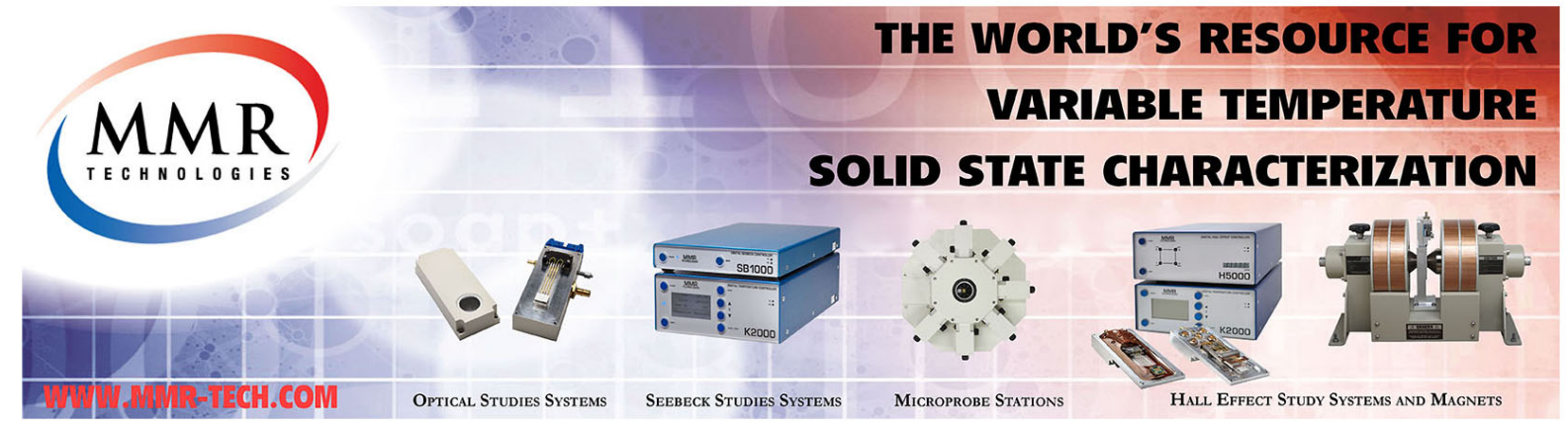




\title{
Thermal expansion in FeCrCoNiGa high-entropy alloy from theory and experiment
}

\author{
Shuo Huang, ${ }^{1, a)}$ Ádám Vida, ${ }^{2,3, b)}$ Wei Li, ${ }^{1}$ Dávid Molnár, ${ }^{1,4}$ Se Kyun Kwon, ${ }^{5}$ \\ Erik Holmström, ${ }^{6}$ Béla Varga, ${ }^{7}$ Lajos Károly Varga, ${ }^{2}$ and Levente Vitos ${ }^{1,2,8, c)}$ \\ ${ }^{1}$ Applied Materials Physics, Department of Materials Science and Engineering, Royal Institute of Technology, \\ Stockholm SE-100 44, Sweden \\ ${ }^{2}$ Institute for Solid State Physics and Optics, Wigner Research Centre for Physics, P.O. Box 49, \\ H-1525 Budapest, Hungary \\ ${ }^{3}$ Department of Materials Physics, Eötvös University, Pázmány Péter Sétány 1/A, H-1117 Budapest, Hungary \\ ${ }^{4}$ Materials Science Group, Dalarna University, 79188 Falun, Sweden \\ ${ }^{5}$ Graduate Institute of Ferrous Technology, Pohang University of Science and Technology, Pohang 37673, \\ South Korea \\ ${ }^{6}$ Sandvik Coromant R\&D, 12680 Stockholm, Sweden \\ ${ }^{7}$ Faculty of Materials Science, Transylvania University of Brasov, Bulevardul Eroilor, \\ No. 29, 500036 Brasov, Romania \\ ${ }^{8}$ Department of Physics and Astronomy, Division of Materials Theory, Uppsala University, Box 516, \\ SE-75120 Uppsala, Sweden
}

(Received 28 April 2017; accepted 1 June 2017; published online 14 June 2017)

\begin{abstract}
First-principle alloy theory and key experimental techniques are applied to determine the thermal expansion of FeCrCoNiGa high-entropy alloy. The magnetic transition, observed at $649 \mathrm{~K}$, is accompanied by a significant increase in the thermal expansion coefficient. The phase stability is analyzed as a function of temperature via the calculated free energies accounting for the structural, magnetic, electronic, vibrational and configurational contributions. The single- and polycrystal elastic modulus for the ferro- and paramagnetic states of the face-centered and body-centered cubic phases are presented. By combining the measured and theoretically predicted temperature-dependent lattice parameters, we reveal the structural and magnetic origin of the observed anomalous thermal expansion behavior. Published by AIP Publishing. [http://dx.doi.org/10.1063/1.4985724]
\end{abstract}

The recently developed high-entropy alloys (HEAs) are composed of more than four principal elements in equal or near equal molar ratios. ${ }^{1,2}$ These alloys have drawn significant attention and opened new exciting research areas with a high intrinsic degree of freedom for designing materials with exceptional properties. ${ }^{3-12}$ Interestingly, even with components having different crystal structures in each ground state, many HEAs were reported to form solid solution phases with face-centered cubic (fcc) or body-centered cubic (bcc) lattices rather than complex intermetallic phases. ${ }^{13,14}$ Aluminum addition to the widely studied FeCrCoNi-based HEA family brings about a structural transition from fcc to bcc with an fcc/bcc duplex region. ${ }^{15-17}$ The structural transition is accompanied by significant changes in mechanical, electrical, and magnetic properties. ${ }^{18-20}$ Similar phenomena were reported in our recent works when adding other sp elements such as $\mathrm{Ga}, \mathrm{Ge}$, and $\mathrm{Sn}$ to the above HEA. ${ }^{21,22}$ Today, very little information is available on the individual alloy phases appearing as a result of crystal-structure engineering. Understanding the intrinsic physical properties of each structure is critical for an optimal tuning of the single- and dual-phase HEAs through microstructure modifications. There exists relatively small number of investigations on this issue because of the complexity of the problem related to the chemical and magnetic disorder in connection with the multicomponent nature of the

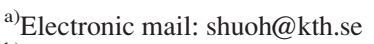

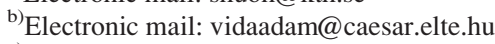

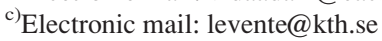

HEAs. In this letter, we employ first-principle alloy theory and key experiments to bring to light the thermal expansion behavior of FeCrCoNiGa HEA adopting an fcc/bcc duplex structure.

The FeCrCoNiGa alloy was prepared from $99.99 \%$ pure elements with induction melting in water-cooled copper crucible. To prevent the sample from oxidation, inert Ar atmosphere was used with titanium getter. The melting process was repeated five times to achieve proper homogeneity of the sample. The chemical analyses of the present alloy were reported in Ref. 21. The Curie temperature was measured with the thermo-gravimetric method (DAM, B60, Ugine Eyraud) in medium vacuum (13 mbar). The heating speed was set as $10 \mathrm{~K} / \mathrm{min}$. The density of the samples was measured with Micromeritics AccuPyc 1340 precision He gas pycnometer, combined with analytical mass measurements. The transversal and longitudinal sound propagation speeds were measured with Olympus $45 \mathrm{MG}$ wall thickness tester. The thermal dilatation was measured with Linseis $\mathrm{L} 75 \mathrm{H}$ equipment, working in medium vacuum mode with applied heating rate of $10 \mathrm{~K} / \mathrm{min}$.

Our ab initio calculations are based on density functional theory ${ }^{23}$ implemented in the framework of the exact muffintin orbitals (EMTO) formalism. ${ }^{24,25}$ In the present application, the chemical and magnetic disorders were treated within the coherent-potential approximation $(\mathrm{CPA}) .^{26-28}$ The oneelectron equations were solved within the scalar-relativistic approximation and soft-core scheme, and the Green functions for the valence states were calculated for 16 complex energy 
points. The muffin-tin basis set included $s, p, d$ and $f$ orbitals. The exchange-correlation effects were treated within the generalized gradient approximation. ${ }^{29}$ The paramagnetic (PM) state was simulated by the disordered local moments (DLM) model. ${ }^{30}$ The validity of this theoretical approach has been verified against observations including the case of $\mathrm{FeCrCoNi}-$ based HEAs. $^{31,32}$

The relative phase stability at ambient pressure and as a function of temperature is investigated from the free energies computed for various structures. Here we decompose the free energy as $F=E+F^{\mathrm{conf}}+F^{\mathrm{mag}}+F^{\mathrm{vib}}+F^{\mathrm{el}}$, where $E$ is the internal energy, and $F^{\text {conf }}, F^{\mathrm{mag}}, F^{\mathrm{vib}}$, and $F^{\mathrm{el}}$ reflect the additional temperature-dependent contributions for the configurational, magnetic, vibrational, and electronic free energies, respectively. For an ideal solid-solution, we have $F^{\text {conf }}$ $=k_{\mathrm{B}} T \sum_{i} c_{i} \ln c_{i}$, where $c_{i}$ is the concentration of atom $i, T$ is the temperature, and $k_{\mathrm{B}}$ is the Boltzmann constant. For the paramagnetic state, the magnetic free energy is estimated within the mean-field approximation as $F^{\mathrm{mag}}=-k_{\mathrm{B}} T \sum_{i} c_{i} \ln \left(1+\mu_{i}\right)$, where $\mu_{i}$ is the local magnetic moment of the $i$ th alloying element. This expression corresponds to a completely disordered paramagnetic state. ${ }^{33}$ The vibrational free energy is derived from the Debye-Grüneisen model $F^{\mathrm{vib}}=9 k_{\mathrm{B}} \theta_{\mathrm{D}} / 8+3 k_{\mathrm{B}} T \ln$ $\left(1-e^{-\theta_{\mathrm{D}} / T}\right)-k_{\mathrm{B}} T D\left(\theta_{\mathrm{D}} / T\right)$, where $\theta_{\mathrm{D}}$ is the Debye temperature, and $D$ is the Debye integral. ${ }^{34}$ Finally, the electronic free energy is defined as $F^{\mathrm{el}}=E^{\mathrm{el}}-T S^{\mathrm{el}}$, where $E^{\mathrm{el}}$ and $S^{\mathrm{el}}$ are the electronic energy and entropy, respectively, which are obtained directly from the EMTO calculations using the finite-temperature Fermi distribution. ${ }^{35}$

Figure 1 shows the calculated temperature-dependent free energies of $\mathrm{FeCrCoNiGa}$ for the fcc and bcc structures at both ferromagnetic (FM) and paramagnetic (PM) states. For clarity, all free energies are plotted with reference to the bcc FM state. It is found that, irrespectively to the crystal structure, the FM state is energetically stable at low temperature, and the PM state becomes favorable at a high temperature. The magnetic transition temperatures are estimated from the present FM and PM free energies to be $643 \mathrm{~K}$ and $691 \mathrm{~K}$ for the fcc and bcc structures, respectively. The Curie temperature $\left(T_{\mathrm{C}}\right)$ obtained from the thermomagnetic measurements is around $649 \mathrm{~K}$. The very good parallelism between the theoretical and experimental $T_{\mathrm{C}}$ values supports the accuracy of the present theoretical scheme. As indicated in Fig. 1, the fcc and bcc phases in the FM state arrive at equilibrium around room temperature where the free energy difference vanishes. Namely, the present system is expected to form a thermodynamic stable fcc-bcc duplex phase near room temperature. This prediction is consistent with experimental observation. ${ }^{21}$ When comparing all four free energies, we find that the FM bcc phase is the most favorable phase at low temperature (cryogenic conditions) and the PM fcc at high temperature. It should be mentioned that samples synthesized in experiments always possess complex microstructures. For example, $\mathrm{FeCr}$-rich precipitates embedded in a NiAl-rich matrix were observed in as-cast $\mathrm{FeCrCoNiAl}$ system. ${ }^{36}$ In the present theoretical model, to better isolate the crystal structure effect, we assumed that the HEAs have perfect composition and display ideal solid solution phase with fcc and bcc lattices, i.e., the ordering/segregation effects were completely neglected. Nevertheless, the good agreement between theory and experiment indicates that the present theoretical approach can capture the structural energy differences and trace energy changes related to the temperature variation in the HEAs.

According to the present $a b$ initio calculations, the lattice vibrations play an important role in the phase stabilization. As the key parameter in the Debye-Grüneisen model, the Debye temperature $\theta_{D}$ can be obtained from the average sound velocity $v_{\mathrm{m}}$ by the relations $\theta_{\mathrm{D}}=\left(\hbar / k_{\mathrm{B}}\right)\left(6 \pi^{2} / V\right)^{1 / 3} v_{\mathrm{m}}$ with $v_{\mathrm{m}}$ $=\left[\left(1 / v_{\mathrm{L}}^{3}+2 / v_{\mathrm{T}}^{3}\right) / 3\right]^{-1 / 3}$, where $\hbar$ is the Planck constant, $V$ is the volume, $v_{\mathrm{L}}$ and $v_{\mathrm{T}}$ are the longitudinal and transverse sound velocities, respectively. The sound velocities are given by the polycrystalline bulk modulus $B$, shear modulus $G$, and density $\rho$, viz., $v_{\mathrm{L}}=\sqrt{(B+4 G / 3) / \rho}$ and $v_{\mathrm{T}}=\sqrt{G / \rho}$. It should be mentioned that the so derived Debye temperatures are consistent with the elastic Debye temperatures obtained by solving the Christoffel equation and the following results are not sensitive to the actual choice. ${ }^{25,37}$ Figure 2 shows the calculated values of $\theta_{\mathrm{D}}, \rho, v_{\mathrm{L}}, v_{\mathrm{T}}$, and $v_{\mathrm{m}}$ for the fcc and bcc $\mathrm{FeCrCoNiGa}$ at both FM and PM states. Our present experimental results are also plotted for comparison. It is found that the theoretical $\rho$ at static conditions $(0 \mathrm{~K})$ is the smallest in the bcc FM state $\left(\sim 8.26 \mathrm{~g} / \mathrm{cm}^{3}\right)$ and the largest in the fcc PM state $\left(\sim 8.38 \mathrm{~g} / \mathrm{cm}^{3}\right)$. These values are only $0.7 \%-2.2 \%$ larger than the experimental value $\left(\sim 8.20 \mathrm{~g} / \mathrm{cm}^{3}\right)$ measured at room temperature. For sound velocity, the calculated $v_{\mathrm{L}}$ and $v_{\mathrm{T}}$ are about $5.37-5.71 \mathrm{~km} / \mathrm{s}$ and $2.69-2.84 \mathrm{~km} / \mathrm{s}$, respectively, which compare well with the corresponding experimental values of $5.22 \mathrm{~km} / \mathrm{s}$ and $2.82 \mathrm{~km} / \mathrm{s}$. Using the computed densities and
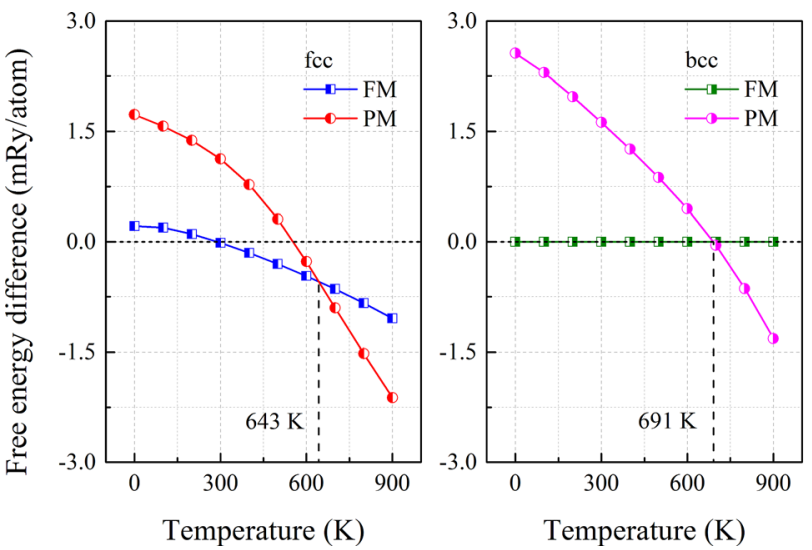

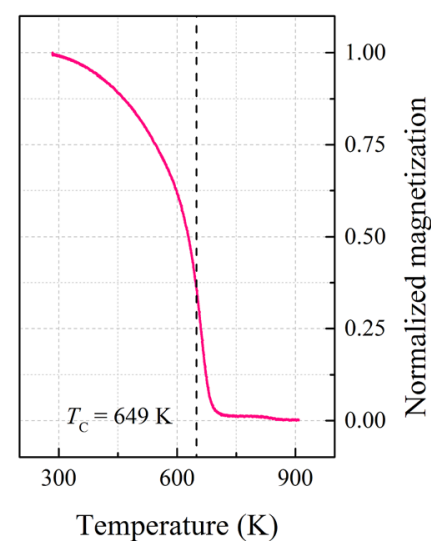

FIG. 1. Temperature-dependent free energies (left and middle panels) of the FeCrCoNiGa high-entropy alloy for the fcc and bcc structures with the ferromagnetic (FM) and paramagnetic (PM) states, respectively. All energies are plotted with respect to the corresponding energy of FM bcc. The right panel shows the measured magnetization after normalization as a function of temperature for the as-cast $\mathrm{FeCrCoNiGa}$ alloy. The dashed line denotes the magnetic transition temperature. 


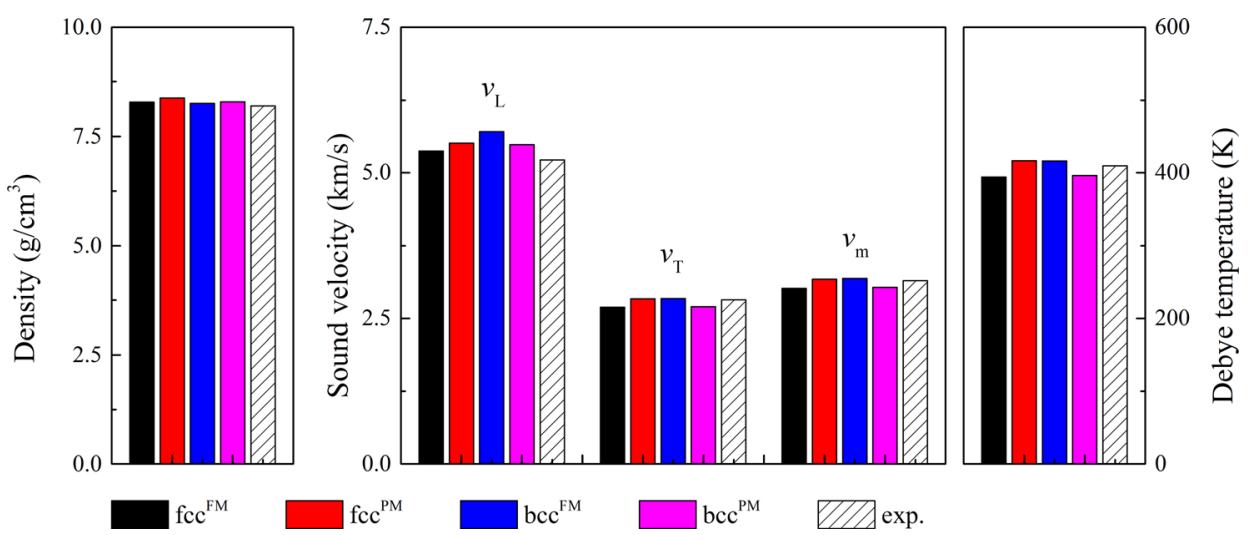

FIG. 2. Density (left panel), sound velocity (middle panel), and Debye temperature (right panel) of the FeCrCoNiGa high-entropy alloy for the fcc and bcc structures with the ferromagnetic (FM) and paramagnetic (PM) states, respectively. The experimental results are shown by the shaded bars. The experimental error bars for the density and sound velocities are $0.2 \%$ and $2.5 \%$, respectively.

sound velocities, for the Debye temperature in the fcc FM (PM) state and bcc FM (PM) state, we get $394 \mathrm{~K}$ (417 K) and $416 \mathrm{~K}(396 \mathrm{~K})$, respectively. These values are very close to $410 \mathrm{~K}$ estimated from measurements.

The corresponding theoretical elastic moduli for the present system are summarized in Table I, along with the available experimental data. Here, the bulk modulus $B$ and its pressure derivative $B^{\prime}$ at equilibrium volume are extracted from the equation of state described by an exponential Morse-type function ${ }^{34}$ fitted to the $a b$ initio total energies for a series of volumes. The two cubic shear modulus $C^{\prime}=$ $\left(C_{11}-C_{12}\right) / 2$ and $C_{44}$ are derived from the volumeconserving orthorhombic and monoclinic deformations. ${ }^{25}$ The polycrystalline shear modulus $G$ is estimated via the arithmetic Hill average $G=\left(G_{\mathrm{V}}+G_{\mathrm{R}}\right) / 2$, where $G_{\mathrm{V}}$ and $G_{\mathrm{R}}$ are the Voigt and Reuss bounds, respectively. ${ }^{38}$ The ratio $A_{\mathrm{VR}}=\left(G_{\mathrm{V}}-G_{\mathrm{R}}\right) /\left(G_{\mathrm{V}}+G_{\mathrm{R}}\right)$ gives an alternative measure of the elastic anisotropy (for isotropic crystal $A_{\mathrm{VR}}$ is zero), which is independent of the crystal structure and thus can be used to compare anisotropies of various structures. The Young's modulus $E$ and Poisson ratio $v$ are connected to $B$ and $G$ by the relations $E=9 B G /(3 B+G)$ and $v=(3 B$ $-2 G) /(6 B+2 G)$, respectively. As shown in Table I, the $G$ and $E$ values for $\mathrm{FeCrCoNiGa}$ are predicted to be 59.9-67.4 GPa and 159.8-178.2 GPa, respectively, which are in line with the corresponding experimental data. ${ }^{21}$ It is interesting to notice that, the present system is expected to be ductile because its $B / G$ ratios in all considered phases are well above the critical value of $1.75 .{ }^{39}$ Furthermore, the PM fcc FeCrCoNiGa is predicted to possess similar ductile/ brittle characteristics as pure Ni based on the Gilman's line $\left(C_{44} / C_{12}\right.$ vs $\left.G / B\right) .^{40}$ The Grüneisen parameter $\gamma$ describes the anharmonic effects and gives the volume dependence of the Debye temperature by $\theta_{\mathrm{D}}(V)=\theta_{\mathrm{D}}\left(V_{0}\right)\left(V_{0} / V\right)^{\gamma}$. We notice that $\gamma$ can be expressed as $\gamma=-f+B^{\prime} / 2$, and for pure metals the factor $f=1 / 2$ gives a good agreement with the experimental data. ${ }^{25,34}$

Figure 3 displays the theoretical lattice parameter $L$ and thermal expansion coefficient $\alpha$ for FeCrCoNiGa in the temperature range of $0-900 \mathrm{~K}$. The values of $L$ at $300 \mathrm{~K}$ are 3.637 (3.627) $\AA$ and 2.885 (2.884) $\AA$ for the FM (PM) fcc and bcc phases, respectively, and they increase by 1.11 (1.33)\% and 0.68 (1.08)\% when the temperature reaches $900 \mathrm{~K}$. It is evident that the temperature gives a larger influence in the fcc phase than in the bcc phase. Further insight can be gained by considering the thermal expansion coefficient defined as $\alpha=(1 / L) d L / d T$. As shown in Fig. 3, we find that the calculated $\alpha$ for all considered phases increases rapidly at low temperatures and gradually turn towards a linear trend at high temperatures. For the highest temperature region considered here, the propensity of increment becomes moderate, especially for the FM bcc. We recall that according to experiments the present system possesses a duplex fcc/bcc structure. ${ }^{21}$ Hence, the actual mean $\alpha$ should be estimated by averaging over the individual phases. For example, the fcc and bcc phases in the FM state are in equilibrium at room-temperature. Assuming equal fractions for the two phases, we get the thermal expansion coefficient $\alpha_{300 \mathrm{~K}} \approx$ $13.3 \times 10^{-6} \mathrm{~K}^{-1}$, which agrees well with the experimental data shown in Fig. 3. The present theoretical value is also close to that of the other HEAs such as FeCrCoNi $\left(\sim 14 \times 10^{-6} \mathrm{~K}^{-1}\right)$ and FeCrCoNiMn $\left(\sim 15 \times 10^{-6} \mathrm{~K}^{-1}\right){ }^{41,42}$ It is of particular interest to highlight that the experimental $\alpha$ increases sharply around the Curie temperature. We notice that theory predicts $\alpha$ values which increase in the order of FM bcc, PM bcc, FM fcc, and PM fcc in the entire temperature range. As a consequence, the fcc/bcc duplex structure of $\mathrm{FeCrCoNiGa}$ in the FM state results in a small $\alpha$ derived as

TABLE I. Theoretical single-crystal elastic constants $\left(C_{11}, C_{12}\right.$, and $C_{44}$, in units of GPa) and polycrystalline elastic modulus $(B, G$, and $E$, in units of GPa), pressure derivative of the bulk modulus $\left(B^{\prime}\right)$, Poisson's ratio $(v)$, Zener anisotropy ratio $\left(A_{\mathrm{Z}}\right)$, Pugh ratio $(B / G)$, and the elastic anisotropy $\left(A_{\mathrm{VR}}\right)$ for the FeCrCoNiGa high-entropy alloy for the fcc and bcc structures and for the ferromagnetic (FM) and paramagnetic (PM) states, respectively. All theoretical results refer to $0 \mathrm{~K}$. The room-temperature experimental shear modulus and Young's modulus are taken from Ref. 21 and they have error bar of $5 \%$.

\begin{tabular}{|c|c|c|c|c|c|c|c|c|c|c|c|c|}
\hline Str. & & $C_{11}$ & $C_{12}$ & $C_{44}$ & $B$ & $G$ & $E$ & $B^{\prime}$ & $v$ & $A_{\mathrm{Z}}$ & $B / G$ & $A_{\mathrm{VR}}$ \\
\hline \multirow[t]{2}{*}{ fcc } & FM & 184.7 & 146.9 & 123.3 & 159.5 & 59.9 & 159.8 & 5.6 & 0.333 & 6.5 & 2.66 & 0.36 \\
\hline & PM & 196.2 & 148.8 & 131.2 & 164.6 & 67.4 & 178.0 & 6.3 & 0.320 & 5.5 & 2.44 & 0.31 \\
\hline \multirow[t]{2}{*}{ bcc } & FM & 215.1 & 162.7 & 122.3 & 180.2 & 66.7 & 178.2 & 4.5 & 0.335 & 4.7 & 2.70 & 0.26 \\
\hline & PM & 193.9 & 156.4 & 125.4 & 168.9 & 60.5 & 162.2 & 5.7 & 0.340 & 6.7 & 2.79 & 0.37 \\
\hline Exp. & & & & & & 65.0 & 168.0 & & & & & \\
\hline
\end{tabular}



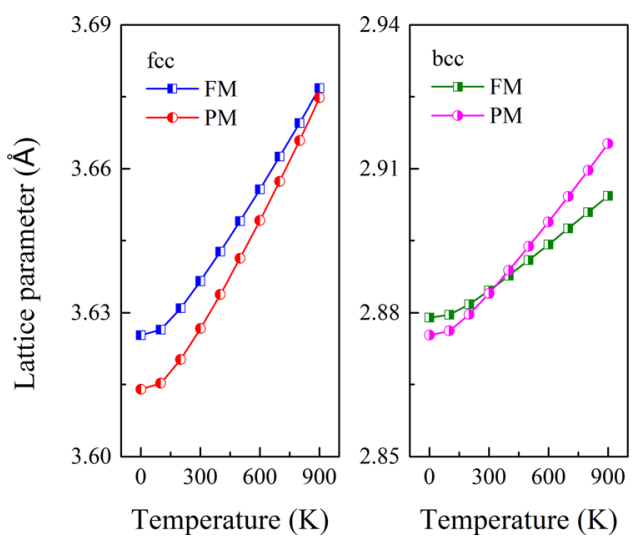

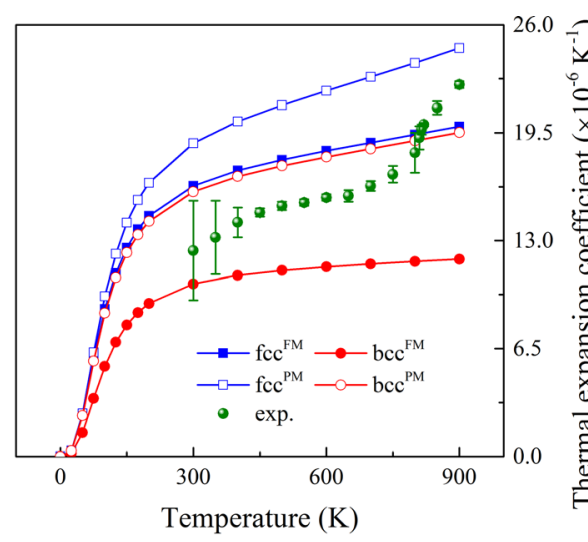

FIG. 3. Lattice parameter (left and middle panels) and thermal expansion coefficient (right panel) of the $\mathrm{FeCrCoNiGa}$ high-entropy alloy for the fcc and bcc structures with the ferromagnetic (FM) and paramagnetic (PM) states, respectively. The experimental results are depicted by circles with error bars. the mean value below the critical temperature. Furthermore, the experimental $\alpha$ is close to the theoretical value obtained for the FM bcc phase at cryogenic conditions and to that for the PM fcc phase at high temperatures. Hence, the sizable difference of the calculated $\alpha$ values between the FM and PM states can explain the observed anomalous thermal expansion behavior.

In summary, our combined experimental and theoretical results demonstrate that the $\mathrm{FeCrCoNiGa} \mathrm{HEA}$ exhibits an anomalous thermal expansion behavior. From the theoretical free energies, a mixture of the fcc and bcc phases is predicted around room-temperature, in good agreement with experiment. The FM bcc phase is energetically the lowest one among the considered phases at cryogenic conditions and the PM fcc phase at high temperatures. The predicted ferro- to paramagnetic transition critical temperatures are close to the measured Curie temperature. In the entire temperature range, the smallest and the largest thermal expansion coefficient occur for the FM bcc phase and PM fcc phase, respectively. The difference between the mean theoretical thermal expansion coefficients below and above the Curie point explains the observed anomalous thermal expansion behavior. The present findings demonstrate that engineering the magnetic and structure transitions provides rich opportunities for designing and optimizing new HEAs with interesting thermophysics properties. Finally, we notice that the revealed strong coupling between these two degrees of freedom brings the present and similar HEAs into the focus of magnetocaloric applications as well.

This work was supported by the Swedish Research Council, the Swedish Foundation for Strategic Research, the Swedish Foundation for International Cooperation in Research and Higher Education, the Carl Tryggers Foundation, the Sweden's Innovation Agency (VINNOVA Grant No. 2014-03374), the China Scholarship Council, the Basic Science Research Program through the National Research Foundation of Korea (NRF) funded by the Ministry of Science, ICT and Future Planning (NRF2014R1A2A1A12067579), and the Hungarian Scientific Research Fund (OTKA 109570). The Swedish National Infrastructure for Computing at the National Supercomputer Centers in Linköping and Stockholm are acknowledged for providing computational facilities.

${ }^{1}$ J. W. Yeh, S. K. Chen, S. J. Lin, J. Y. Gan, T. S. Chin, T. T. Shun, C. H. Tsau, and S. Y. Chang, Adv. Eng. Mater. 6, 299 (2004).
${ }^{2}$ B. Cantor, I. T. H. Chang, P. Knight, and A. J. B. Vincent, Mater. Sci. Eng. A 375-377, 213 (2004).

${ }^{3}$ J. W. Yeh, Ann. Chim. Sci. Mater. 31, 633 (2006).

${ }^{4}$ Y. Zhang, T. T. Zuo, Z. Tang, M. C. Gao, K. A. Dahmen, P. K. Liaw, and Z. P. Lu, Prog. Mater. Sci. 61, 1 (2014).

${ }^{5}$ Y. F. Ye, Q. Wang, J. Lu, C. T. Liu, and Y. Yang, Mater. Today 19, 349 (2016).

${ }^{6}$ Z. Li, K. G. Pradeep, Y. Deng, D. Raabe, and C. C. Tasan, "Metastable high-entropy dual-phase alloys overcome the strength-ductility trade-off," Nature 534, 227-230 (2016).

${ }^{7}$ Y. Zou, H. Ma, and R. Spolenak, Nat. Commun. 6, 7748 (2015).

${ }^{8}$ B. Gludovatz, A. Hohenwarter, D. Catoor, E. H. Chang, E. P. George, and R. O. Ritchie, Science 345, 1153 (2014).

${ }^{9}$ P. Koželj, S. Vrtnik, A. Jelen, S. Jazbec, Z. Jagličić, S. Maiti, M. Feuerbacher, W. Steurer, and J. Dolinšek, Phys. Rev. Lett. 113, 107001 (2014).

${ }^{10}$ K. Y. Tsai, M. H. Tsai, and J. W. Yeh, Acta Mater. 61, 4887 (2013).

${ }^{11}$ O. N. Senkov, G. B. Wilks, D. B. Miracle, C. P. Chuang, and P. K. Liaw, Intermetallics 18, 1758 (2010).

${ }^{12}$ Y. J. Zhou, Y. Zhang, Y. L. Wang, and G. L. Chen, Appl. Phys. Lett. 90, 181904 (2007).

${ }^{13}$ B. S. Murty, J. W. Yeh, and S. Ranganathan, High Entropy Alloys (Butterworth-Heinemann, 2014).

${ }^{14}$ M. C. Gao, J. W. Yeh, P. K. Liaw, and Y. Zhang, High-Entropy Alloys: Fundamentals and Applications (Springer, Switzerland, 2016).

${ }^{15}$ H. P. Chou, Y. S. Chang, S. K. Chen, and J. W. Yeh, Mater. Sci. Eng., B 163, 184 (2009).

${ }^{16}$ W. R. Wang, W. L. Wang, S. C. Wang, Y. C. Tsai, C. H. Lai, and J. W. Yeh, Intermetallics 26, 44 (2012).

${ }^{17}$ Y. F. Kao, T. J. Chen, S. K. Chen, and J. W. Yeh, J. Alloys Compd. 488, 57 (2009).

${ }^{18}$ Y. F. Kao, S. K. Chen, T. J. Chen, P. C. Chu, J. W. Yeh, and S. J. Lin, J. Alloys Compd. 509, 1607 (2011).

${ }^{19}$ W. R. Wang, W. L. Wang, and J. W. Yeh, J. Alloys Compd. 589, 143 (2014).

${ }^{20}$ S. Huang, W. Li, X. Li, S. Schönecker, L. Bergqvist, E. Holmström, L. K. Varga, and L. Vitos, Mater. Des. 103, 71 (2016).

${ }^{21}$ Á. Vida, L. K. Varga, N. Q. Chinh, D. Molnár, S. Huang, and L. Vitos, Mater. Sci. Eng. A 669, 14 (2016).

${ }^{22}$ S. Huang, Á. Vida, D. Molnár, K. Kádas, L. K. Varga, E. Holmström, and L. Vitos, Appl. Phys. Lett. 107, 251906 (2015).

${ }^{23}$ P. Hohenberg and W. Kohn, Phys. Rev. 136, B864 (1964).

${ }^{24}$ L. Vitos, Phys. Rev. B 64, 014107 (2001).

${ }^{25}$ L. Vitos, Computational Quantum Mechanics for Materials Engineers (Springer, London, 2007).

${ }^{26}$ P. Soven, Phys. Rev. 156, 809 (1967).

${ }^{27}$ B. L. Győrffy, Phys. Rev. B 5, 2382 (1972).

${ }^{28}$ L. Vitos, I. A. Abrikosov, and B. Johansson, Phys. Rev. Lett. 87, 156401 (2001).

${ }^{29}$ J. P. Perdew, K. Burke, and M. Ernzerhof, Phys. Rev. Lett. 77, 3865 (1996).

${ }^{30}$ B. L. Győrffy, A. J. Pindor, J. Staunton, G. M. Stocks, and H. Winter, J. Phys. F: Met. Phys. 15, 1337 (1985).

${ }^{31}$ F. Y. Tian, L. Delczeg, N. X. Chen, L. K. Varga, J. Shen, and L. Vitos, Phys. Rev. B 88, 085128 (2013).

${ }^{32}$ S. Huang, W. Li, S. Lu, F. Tian, J. Shen, E. Holmström, and L. Vitos, Scr. Mater. 108, 44 (2015).

${ }^{33}$ G. Grimvall, Phys. Rev. B 39, 12300 (1989).

${ }^{34}$ V. L. Moruzzi, J. F. Janak, and K. Schwarz, Phys. Rev. B 37, 790 (1988). 
${ }^{35}$ L. Vitos, P. A. Korzhavyi, and B. Johansson, Phys. Rev. Lett. 96, 117210 (2006).

${ }^{36}$ A. Manzoni, H. Daoud, R. Völkl, U. Glatzel, and N. Wanderka, Ultramicroscopy 132, 212 (2013).

${ }^{37}$ G. Grimvall, Thermophysical Properties of Materials (North-Holland, Amsterdam, 1999).

${ }^{38}$ R. Hill, Proc. Phys. Soc. Sect. A 65, 349 (1952).
${ }^{39}$ S. F. Pugh, Philos. Mag. 45, 823 (1954).

${ }^{40}$ J. J. Gilman, Electronic Basis of the Strength of Materials (Cambridge University Press, Cambridge, 2003).

${ }^{41}$ K. Jin, S. Mu, K. An, W. D. Porter, G. D. Samolyuk, G. M. Stocks, and H. Bei, Mater. Des. 117, 185 (2017).

${ }^{42}$ G. Laplanche, P. Gadaud, O. Horst, F. Otto, G. Eggeler, and E. P. George, J. Alloys Compd. 623, 348 (2015). 